\title{
TRAINING CONSIDERATIONS FOR EXPERT PILOT DECISION MAKING
}

Richard J. Adams

Advanced Aviation Concepts, Inc. and

\author{
Ronald John Lofaro, Ph.D. \\ Federal Aviation Administration
}

\begin{abstract}
Extensive research since 1977 by the Federal Aviation Administration determined that the predominant underlying cause of "pilot error" accidents involved decisional problems or cognitive information processing. To attack these problems, Aeronautical Decision Making (ADM) training materials were developed and tested for ten years. Following this development, twelve training manuals were published covering the spectrum from student/private pilots, instrument pilots and commercial airline crews. The publication and use of these manuals has had a dramatic effect on the reduction of human performance error related accidents. These successes are documented for civil and military operations both in the U.S. and worldwide. However, shortcomings have been observed in the use of the ADM training for recurrency and in their relevance for more experienced pilots. This research identifies the differences between expert and novice decision makers from a cognitive information processing perspective and correlates the development of expert pilot cognitive processes with training and experience. This introductory material should provide an understanding of how to formulate expert pilot decision making training innovations and how to continue the record of improved safety through ADM training.
\end{abstract}

\section{Introduction}

Decision making training has made a significant impact on safety by reducing the human performance error related accidents in both civil and military aviation. Accident rate reductions of about $50 \%$ can be seen when comparing pilot groups with and without decision making training (Diehl, 1991). Yet, these large safety improvements have not reached the entire pilot community. In particular, there is a lack of acceptance of the linear decision making model by the more experienced pilots, a resistance to change in interpersonal skills in the multicrew environment and even a "boomerang" effect where the attitude toward the use of all crew resources deteriorates after training.

Extensive research and empirical testing in Aeronautical Decision Making (ADM) produced a series of ten Federal Aviation Administration manuals and reports on ADM (1986-1988). Although it is admittedly difficult to accurately assess the impact of all of the manuals throughout aviation,

*This report is based, in part, on research supported by the U.S. Department of Transportation Federal A viation Administration Contract Number DTFA01-90-C-00042. The content of this report reflects the views of the authors and not necessarily those of the FAA or any of its organizational entities. 
significant reductions in human performance error (HPE) accidents have been documented in specific areas:

-A 36\% reduction in all HPE accidents for the worldwide B206 fleet (Fox, 1991).

-A $72 \%$ reduction in weather related HPE accidents for the worldwide B206 fleet (Fox, 1991).

-A 48\% reduction in all HPE accidents for the U. S. B206 fleet (Fox, 1991).

-A $54 \%$ reduction in HPE accidents for the largest U.S. civil helicopter operator (more than two million takeoffs and landings annually, Fox, 1991)

- A $51 \%$ reduction in accidents $/ 100,000$ hours for USAF MAC crews (Diehl, 1991).

- A 20\% reduction in USN helicopter air crew mishap rate and an $\mathbf{8 1 \%}$ reduction by $\mathrm{A} 6$ and EA6 airplane pilots (Alkov, 1991)

These findings generated a request from many in the aviation community for advanced decision making and crew resource management material. They also have led the National Transportation Safety Board to recommend that the FAA pursue the implementation of ADM more vigorously following a fatal 1991 accident involving an airplane and a helicopter (NTSB 1991) However, as dramatic as the examples of improvements were, a more detailed examination of the accident rate reduction data disclosed that the major positive impact has been on the less experienced pilots (Albert, 1989). This finding led to two questions: Can we achieve the same impact in human error reduction with more experienced pilots? And, how can this be done?

The current research effort attempts to respond to these questions, questions which industry has also asked. The research is based upon parallel events occurring in the air carrier industry during the 19831989 timeframe. During this period, there were several extraordinary accidents involving multiple engine failures, explosive decompressions caused by structural failures, fuel starvation and in-flight fires. In each of these accidents, experienced pilots quickly responded to emergencies for which there was no handbook procedure or previous training. They assessed the situation and integrated airmanship skills, trained procedures and aeronautical knowledge into a quick, effective decision making process. Such dynamic cognitive behavior was in direct contrast to the more basic ADM training which stressed a linear, measured approach to situation analysis.

\section{Expert Cognitive Processes}

Research in the last twenty years has revealed that superior performance is most often the result of the interaction between accumulated skill and experience. The primary differences between a beginner and an expert can be attributed to acquired knowledge and problem solving skills: what we call expertise and which is demonstrated through performance. Expert performance can be defined as the selection of an appropriate response to situations or problems in a wide variety of domains. These include selecting the best move in a chess game, correctly diagnosing a medical problem, or using the proper emergency procedure in aviation. The relevant research on expert performance has focused on the basic understanding of knowing how to do something well rather than knowing what the underlying mechanism was for superior performance (Dreyfus \& Dreyfus, 1986). Complex problem solving research assumed that the integration of the basic human information processing skills was required. This included the processes of perception, memory, attention, and reasoning. This research had real-world importance since performance/expertise obviously depended on learning how to do something well. 
All human cognition is task dependent and purposeful (goal oriented). Humans use their knowledge, cognitive processing skills and the cues or stimuli of a situation or task to develop problem solving approaches. To accomplish this, two types of knowledge are used (Anderson, 1985). These are declarative knowledge and procedural knowledge. Declarative knowledge consists of knowledge that can be verbalized, some say knowledge about "facts and things". Procedural knowledge is knowledge about actions or how to perform various cognitive activities. These ordina rily cannot be completely or adequately verbalized, for example, how to ride a bike. Procedural knowledge is the basis for development of specific steps (also called production rules) to be used in problem solving situations. The study of procedural learning became a crucial area to be understood. The current (general) theory of acquiring expertise includes the following three stages:

1. Novice's solve problems by weak, domain general, heuristic methods (often working backwards from the goal).

2. Successful solutions (when repeated frequently) lead to the development of domain specific procedures or production rules. These rules specify actions that will achieve goals under particular conditions. Production rules form the beginnings of expertise.

3. As these rules are used more and more often, and applied to many situations in a domain, they result in fairly automatic generation of specialized productions which often use forward inferencing to progress from the initial problem state toward a solution or goal. Thus, relative to the novice, the expert is able to reach the correct solution more quickly and efficiently.

The status of these theories of expertise are presented in two references which provide 24 "Summary Propositions" pertinent to aviation. Thoughts on Expertise (Glaser, 1987) and On the Nature of Expertise (Glaser and Chi, 1988) provide the following relevant findings:

1. Expert performance is characterized by rapid access to a well organized body of conceptual and procedural knowledge. High levels of competence result from the interaction between knowledge structure and processing abilities.

2. The organization of knowledge used by experts can be thought of as schemata or a modifiable information structure based upon knowledge that is experienced. Schema theory assumes there are schemata for recurrent situations that expedite decisions in certain situations.

3. Expertise is domain specific. Within a domain, experts develop the ability to perceive large meaningful patterns. Furthermore, the expert's pattern recognition occurs so rapidly that it appears to take on the character of insight or intuition.

4. Expert knowledge is highly procedural and goal oriented. Individuals with extensive domain knowledge are much better at relating events in cause-andeffect sequences that relate to the goals and subgoals of a problem solution.

5. The capability of experts to fast-access their knowledge facilitates problem perception in a way that leads to the reduction of the role of memory search and general processing. The outstanding performance of experts is derived from how their knowledge is structured to accomplish: Retrieval, Pattern Recognition, and Inference. 
6. Generalized thinking and problem solving skills may develop in individuals who acquire expertise in several domains (e. g., aeronautics, airplane systems, air traffic control procedures, emergency procedures, etc). Continuous development of expertise in a field is based upon novel conditions that extend competence to novel situations.

7. Experts develop specialized schemata that match goals to demands of the problem. Although both novices and experts can display good use of general problem solving process, experts use them primarily in unfamiliar situations.

8. The development of expertise is influenced by task demands encountered in the course of experience. In some domains, experts develop the capability for "opportunistic planning" which enables them to revise problem representations and to access multiple possible interpretations of a situation.

9. Experts also develop types of metacognition or self-regulatory capabilities that are not present in less experienced decision makers. Experts' skilled self-regulatory processes free their working memory for higher level conscious processing. These include: planning ahead, efficiently monitoring one's time and attentional resources, and monitoring and editing one's efforts to solve a problem.

10. An important point of distinction is that there are both routine and adaptive experts. Adaptive experts possess the ability to creatively respond to novel situations and develop an appropriate response with some reasonable chance for a successful outcome.

This distinction between routine and adaptive experts leads to the threshold of the next generation of expertise theory which relies on a cognitive psychology perspective. The current FAA sponsored R\&D will examine applications to real world problems and the focus will be on how aviators respond to untrainable emergencies. A broad distinction between two classes of expertise is suggested in Sloboda (1991). His definition is that expert performance involves "the reliable attainment of specific goals within a specific domain." An extended definition is that "an expert is someone who can make an appropriate response to a situation which contains a degree of unpredictability."

In general, an expert will succeed in identifying and adapting to the inherent constraints of the task. If the task can be done most efficiently by forward search, the expert will search forward; if backward search is better, the expert searches backward. If certain patterns of cues are crucial to performing the task well, the expert will likely perceive and remember them; if patterns are not so important, the expert will not selectively process them.

\section{EXPERTISE AND TRAINING OR PRACTICE}

Initially, expert performance and expertise involves the development of encoding processes which allow the situation to be fully represented and integrated cognitively. In this way, relevant actions can be retrieved from memory. The internal representation of external situations is also critical to planning and evaluation of possible courses of action as well as a means to represent a dynamically changing environment for the purposes of anticipation and prediction.

It seems that acquisition of expertise can be increased for most, if not all, relevant aspects of performance. Table 1 shows a phase or stage view of differing levels of expertise. 
Table 1 PHASES AND CATEGORIES OF EXPERTISE

\section{PHASES OF EXPERTISE}

Beginning Phase (Acquisition of declarative knowledge and domain general problem solving skills)

About 1-2 years of active experience and training

Many years of active experience and training

(Full time - 40-80 hours per week)

More than 10 years of full time experience and training

\section{CATEGORY OF EXPERT}

Beginner, Student, or

Novice

Intermediate

Routine Expert

(or "Journeyman")

Master or Adaptive Expert

At this time, knowledge about how experts attain the base for their expert performance is relatively limited. Generally speaking, the current view is that the novice should have acquired all basic knowledge in less than one year. Continuing beyond this basic knowledge leads to the acquisition of problem solving skills where the knowledge is organized to effectively produce efficient performance. That is, there is an acquisition of the procedural knowledge of complex patterns occurring in specific situations. At this Intermediate level, differences in expertise appear to be related to the cued recall ability and the number and complexity of those patterns available for use. Finally, in both the routine expert and adaptive expert categories, an accepted, domain specific vocabulary (or jargon) is developed to allow efficient communication among experts in a given domain. This is obvious in aircraft operations (from flight planning to air traffic control) where experts have developed an extensive jargon which is formalized in the "Pilot-Controller Glossary" of the Airman's Information Manual.

\section{Pilot Cognition \& Information Processing}

Thus far, we have characterized the performance of experts from a cognitive psychology perspective. We have tried to show that the development of expertise relies heavily on training and requires considerable amounts of experience in a specific field. Further, experts rely on a wide variety of different processing skills and unique problem solving capabilities. As summarized in Gordon (1990):

- Experts have more detailed, better organized knowledge structures.

- Experts perceive and organize problems on a more abstract level than novices.

- Experts perceive problems in large meaningful patterns related to the context.

- Experts are much faster than novices because of their use of procedural knowledge and forward inferencing techniques.

These characteristics are equally applicable to the expert pilot domain and all have been observed and documented. We will now attempt to show how the cognitive psychology perspectives and understanding of the development of expertise apply to pilot development, training and aeronautical decision making. 
Cognitive psychology recognizes three stages in the development of expert problem solving skills (Anderson 1985). These are cognitive, associative and autonomous. During the first, cognitive stage, pilots commit to memory a set of facts relevant to a desired skill. They typically rehearse these facts as they first perform the skill. For example, novice pilots learning stall recovery will memorize: recognize the stall, lower the nose, apply full power, level the wings and minimize altitude loss. In this stage, they are using their general aeronautics knowledge (domain-general) to guide their solution to loss of lift over one wing, and solve a domain specific problem, how to keep the aircraft flying. The problem solving capabilities and level of expertise in this stage are very basic. Novices spend a lot of time searching and moving around factual knowledge.

The second, or associative stage, has two important characteristics. First, errors in the initial understanding and performance are detected and gradually eliminated. That is, the novice pilot learns to coordinate the nose drop, power application and rudder application for a smooth stall recovery. Second, the connections between the various elements required for successful performance are strengthened. The pilot does not sit for a few seconds trying to decide which action to perform first after lowering the nose. Basically, the outcome of the associative stage is a learned procedure for performing a desired response to a known situation.

The third cognitive stage occurs when the problem solving procedures become faster and more automatic. The autonomous stage evolves from the repeated application of known patterns and their associative use to achieve solutions. The use of declarative knowledge or "verbal mediation" often disappears during this stage of cognitive processing, at least for some tasks. Expert cognitive process development gradually improves in a specific area or domain. Ultimately, the skill can be extended to the ability to respond to cues not previously encountered and to develop new solutions or production rules applicable to novel situations.

In aviation, training is highly procedure oriented both in developing flying skills (psychomotor) and in decision making skills (cognitive and informational) for normal and emergency operation of the aircraft. These procedures and skills provide the foundation for the development of more sophisticated production rules (procedural knowledge) as experience is gained. Novice aviators develop flying and decision making skills through 1-5 years of experience. This experience allows pilots to expand their procedural knowledge base using encounters with real-world problems and operational constraints. The low time pilot is at the second stage of cognitive process development; he has begun to develop the speed and quality of processing of the Routine Expert. Finally, the Expert Pilot mainly relies on automatic cognitive processing abilities. Just as in the other domains of sports, games, music, and medicine, the Expert Pilot has achieved a tremendous base of procedural knowledge and skills applicable to normal day-to-day flying problems, trained emergencies (such as an engine failure) and novel or untrainable emergencies.

Figure 1 illustrates the relationships between levels of pilot experience, types of knowledge used for problem solving and the three stages of development of cognitive processing ability. As shown in the figure, one main characteristic of the development of expert cognitive processes is the continual increase in decisional speed and accuracy as experience is gained in a specific area, e.g., aviation. In fact, these two characteristics are precisely the areas of decision making and problem solving most affected by experience and training or "practice." 


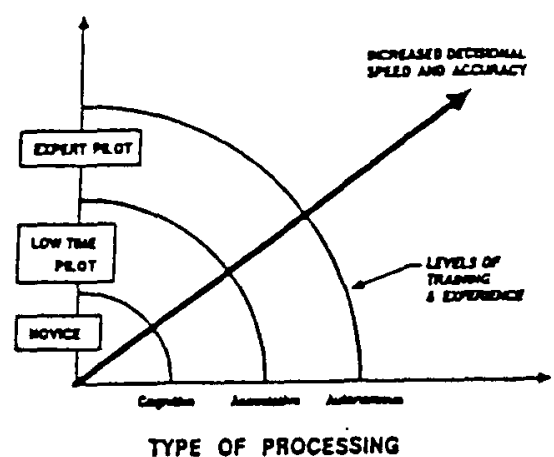

Figure 1 EXPERT PILOT JUDGMENT DEVELOPMENT

To summarize, novice pilots respond (cognitively) to stimuli or external cues based upon an understanding of a complex, declarative knowledge base. Their decisions, whether normal or critical, are typically based on a linear problem solving approach (some type of checklist). Their capabilities are generally limited to the procedures learned and expedited by the use of rules-of-thumb (or heuristics). The intermediate pilot is becoming an associative problem solver and has the capability for an enhanced decision making. As a result of experience and additional flight training, this pilot has the capacity for more dynamic cognitive processing. At the associative level, pilots store information in terms of schemata which are modifiable information structures based upon experience. This "associative pilot" uses pattern recognition and dynamic interrelationships among objects, situations and events to integrate and interpret related knowledge instead of the static, linear thinking of the novice. This pilot's level of cognitive processing is in the process of evolving into a Routine expert.

In addition to having all the decision making skills gained through experience and training, the Expert Pilot is "adaptive." Expert's can alter procedures in real time (modify, delete or expand); can create new rules and patterns based upon unique, previously unencountered problem characteristics. This capability to creatively respond to unique problems or novel task demands identifies the highest level of expert pilot cognitive processes.

This "adaptive" capability is referred to as "KNOWING WHEN" (Dreyfus and Dreyfus, 1986). That is, the Adaptive Expert Pilot can perceive the necessity to alter ingrained procedures based upon the parameters and dynamics of the problem or situation encountered. When necessary, the expert is able to plan and set goals required to accomplish a successful solution. It is believed that this "KNOWING WHEN" (an almost direct perception of the proper course of action) may provide the key to the next generation of ADM training. As in the general field of expertise, isolating and quantifying the cues that experts use to either trigger a routine response or the mechanism to adapt remains a challenge.

\section{Training Considerations}

Two issues must be considered when teaching decision making to experienced pilots. These are nonlinear decision making and cognitive dissonance.

Non Linear Decision Making: Currently there are a large number of both competing and complementary decision making models and procedures (Maher, 1991). A few of these either were aviation developed, 
modified for aviation use or applied to aviation. These include the DECIDE model; the PASS model; and, the SAFE model. However, no definitive research exists which allows for the identification of one optimum decision making theory - either for the pilot or crew. All theories have positive aspects and drawbacks; all have difficulty in meeting all the unique and stringent requirements that aviation imposes. However, a great deal is known about establishing and promoting a set of environmental conditions which foster optimal crew decisional processes and strategies (Lofaro, 1992).

One of the newest areas that holds promise for the development of ADM comes from Mathematics/Artificial Intelligence; it is Chaos theory (Gleick, 1988). All current models of ADM are essentially linear. Some models have branching (decisional "trees") aspects, but all involve a linear series of steps/choices. The causal chain model used in accident analysis is an example of an essentially linear view of a complex event. It is true that a linear chain of prior events can be reconstructed for an accident. But, such a chain is not sensitive to the fact that small changes (in the environment, in time-pressure, in the crew composition, etc.) can make large--and unpredictable by any linear model-- differences in the actions and consequences as time passes.

Since many ADM models are based on accident analysis (on breaking the causal chain), once again the linear DM paradigm is used. It is becoming apparent that, especially under time pressure, we make decisions in a non-linear fashion; this holds also for group decisions (Lofaro, 1991). Another short-fall of ADM models based on accident data is that they rely on an analysis of one (or a statistical representation of many) prior event(s). They then are either so general as to not be helpful in a particular situation or so complex and specific that they only apply to one situation which will never re-occur in exactly the same way. Chaos theory deals with non-linear systems and the corresponding beginnings of the realization that the human mind does not typically use linear steps to decide. Rather, cognitive processes go forward and backward, sideward and into many layers simultaneously. Insight and direction for expert decision making training may be available from this field.

Cognitive Dissonance. The psychological phenomena of cognitive dissonance may, in part, help to explain why some high-time aviators show less acceptance of ADM --- as well as less attitudinal change after exposure to ADM and/or CRM training materials. An analogy may clarify this statement:

Consider if you will the baseball, a small hard core wrapped with layers of varying types of twine and covered with an outer shell of stitched-on horsehide. The core is well protected by the layers of twine and the cover. The twine layers themselves are of different strength and they may be wound or wrapped with more or less tension. The core can be considered our basic, deepest values and beliefs; the twine windings are less tightly held beliefs and attitudes; and, the cover is what holds it all together and what must be penetrated to access the interior of the baseball--- the biases and slants and filters by which we initially process new data.

Following this analogy, it would seem that the attitudes and beliefs of high time aviators are closer to the core and therefore less amenable to change. Or, looked at another way, their flying habits and attitudes are more embedded, therefore, challenges may trigger a cognitive dissonance based "protection". The initial reaction of such pilots to ADM information and strategies which run counter to their own are typical cognitive dissonance mechanisms by which people do not change their attitudes and beliefs when confronted with new, unsettling data. 
Typically, the person experiencing cognitive dissonance responds by challenging or rejecting the data which has caused the dissonance. This is done by forgetting it, by questioning the source, and/or by finding others of similar beliefs and attitudes. This serves to reinforce the original beliefs and attitudes of all concerned. This can be a partial explanation of what Dr. Robert Helmreich has found in some CRI $A$ trainees and what he terms the "boomerang effect" (Helmreich, 1989). Dr. Helmreich has found that some pilots receiving CRM training not only resist attitudinal change, but also experience either a hardening or "negative increase" of their initial attitudes, as well as sometimes attempting to proselytize others in the CRM class.

\section{Conclusions}

A review of aviation examples where expert pilots "saved the day" either in whole or in part, documented that pilot's making decisions under stress exhibit five basic characteristics (Adams and Ericcson, 1992):
Reversion to basic airmanship skills
Instantaneous recall of training
Reasoned approach in emergencies
Positive in approach \& expectations
Self-assured and optimistic.

This research effort has identified the characteristics of expert pilot decision making and identified the differences between expert and novice pilot cognitive processing skills. This is the initial step in the development of expert pilot decision making training. However, additional research is required in three areas to further our understanding of how the adaptive expert pilot functions and how to train novice and intermediate pilots this "adaptiveness."

The first area is to acquire a better understanding of the adaptive expert's perception of information and the decision related actions. This can be done by analysis of the different interpretations of task demands between novice and expert pilot's when faced with the same cues and context, $i$. e., their sensing and filtering cognitive processes. This analysis should also include a more detailed examination of differences between novice and expert pilot's in setting goals and taking action on the available information.

The second task should be a closer examination of how experienced pilot's have applied their cognitive abilities in both trainable and untrainable emergency situations. This empirical data base coupled with current efforts in modeling chaotic systems and the importance of knowing when to "adapt" cognitive processing to meet novel task demands may provide enough information to postulate an initial expert pilot decision making model.

The third area to explore is the importance and impact of cognitive dissonance in experienced pilot decision making training. An analysis of the possible differences in the expert and novice psychophysiological attitudes including kinesthetic, affective and cognitive components should be performed. The importance of affective components and characteristics in problem solving should be analyzed to determine what relationships might exist between these characteristics and decision making. These issues should be addressed in expert decision making training which provides analogous affective states to reinforce the development of analytical relations between the training environment and what is perceived as the operational environment. 
If a new level of understanding of expert pilot cognitive processes in these three areas can be achieved, then safety could be further improved through more advanced, tailored training. Again, teaching judgment or decision making skills (of a more advanced nature) avoids the pitfalls of learning totally from the expensive school of accident/incident experience.

\section{References}

Adams, R. J. and Ericsson, K. A., (1992). Cognitive processes in expert pilot decision making. Advanced A viation Concepts, Inc., for the Federal Aviation Administration. Report No. DOT/FAA$\mathrm{PM} / 12$.

Adams, R. J. and Weiss, R. W. (1991). Enhanced training to reduce pilot errors. 46th Annual Forum Proceedings. American Helicopter Society.

Albert, V. E. (1989). Keynote speech at HELIPROPS advisory committee meeting. Helicopter Association International Annual Meeting. New Orleans, LA.

Alkov, Robert A., (1991) U. S. Navy air crew coordination training - A progress report. U. S. Naval Safety Center for Sixth International Symposium on A viation Psychology. The Ohio State University, Columbus, $\mathrm{OH}$.

Anderson, J. R. (1985). Cognitive psychology and its implications. W. H. Freeman and Company, New York. Chi, M., Glaser, R., \& Farr M. (eds.) (1988). On the nature of expertise. Hillsdale, NJ: Erlbaum.

Diehl, A. E., (1991). The effectiveness of training programs for preventing aircrew error. U. S. Air Force Inspection and Safety Center for the Sixth International Symposium on Aviation Psychology, The Ohio State University.

Dreyfus, H. L. and Dreyfus, S. E. (1986). Mind over machine. The Free Press, New York, N. Y.

Fox, Roy G.,1991). Measuring safety in single-and twinengine Helicopters. In the Flight Safety Digest. Flight Safety Foundation: Alexandria, VA.

Glaser, R. (1987). Thoughts on expertise. In C. Schooler \& W. Schaie (eds.). Cognitive functioning and social structure over the life course (pp. 81-94). Norwood, NJ: Ablex Publishing Corp.

Gleick, J. (1988). Chaos making a new science. Penguin Books: New York, N. Y.

Gordon, S. E. Implications of cognitive theory for knowledge acquisition. Chapter to be published in - The cognition of experts: Psychological research and empirical AI. Hoffman, R. (ed.), in press.

Helmreich, R. L. and Welhelm, J. A., (1989). When training boomerangs: Negative outcomes associated with CRM programs Proceedings of the Fifth International Symposium on Aviation Psychology, The Ohio State University Press, Columbus, Ohio, April 1989.

Lofaro, R. J. (1992). Crew resource management: Past, present and future. The Journal of Aviation/Aerospace Education \& Research, in press.

Lofaro, R. J. (1991). Crew resource management integration. Presentation at the joint ATA/FAA Workshop on Integrated CRM, Washington, D. C., 19-21 November 1991.

Maher, J. W. (1991). Why pilots are least likely to get good decision making when they need it most. Proceedings of the Sixth International Symposium on Aviation Psychology, The Ohio State University Press, Columbus, Ohio.

National Transportation Safety Board, (1991). Aviation Accident Summary Report - Mid-Air Collision between a Lycoming Air Services Piper Aerostar and a Sun Oil Company Bell 412. Marion, Pennsylvania, April 1991, NTSB 91-27.

Sloboda, J. (1991). Musical expertise. In K. A. Ericsson and J. Smith (eds.) Toward a general theory of expertise: Prospects and limits. New York: John Wiley. 